Check for updates

Cite this: RSC Adv., 2017, 7, 37459

Received 29th April 2017 Accepted 23rd July 2017 DOI: 10.1039/c7ra04814a

rsc.li/rsc-advances

\section{Metabolite identification and pharmacokinetic study of platycodi radix (Jiegeng) in vivo $\dagger$}

\author{
Zhongyao Tang, ${ }^{a}$ Yuanyuan Hou, (D) *a Xueyan Hu, ${ }^{a}$ Aina Liu, ${ }^{a}$ Leefong Yau, ${ }^{\text {b }}$ \\ Tiantian Tong, ${ }^{\mathrm{b}}$ Zhihong Jiang $\mathbb{D D}^{\mathrm{b}}$ and Gang Bai ${ }^{\mathrm{D}}{ }^{\mathrm{a}}$
}

Platycodi radix (Jiegeng) is commonly used as a channel ushering drug for assistance in treating respiratory diseases, because of the activities of saponins that are called platycosides. In this study, using ultra high pressure liquid chromatography with quadrupole time of flight mass spectrometry (UPLC-Q/TOF-MS) and a strategy based on fragmentation behaviors, a total of thirteen platycosides were identified in the total saponins, and seven of these were found in rat plasma following a single oral dose of total saponins. The secondary platycosides were proved to be the main absorbed saponins in vivo. The major absorbed platycosides, 3-O- $\beta$-D-glucopyranosylplatyconic acid (GPA) and 3-O- $\beta$-Dglucopyranosylplatycodigenin (GP), were isolated from total saponins and the pharmacokinetics were evaluated in mice by liquid chromatography with triple quadrupole mass spectrometry (LC-QQQ). The results of the pharmacokinetic analysis indicated that GPA and GP were quickly absorbed with short $T_{\max }$ (<45 min). $C_{\max }\left(\mu \mathrm{g} \mathrm{L}^{-1}\right), \mathrm{AUC}_{0-t}\left(\mu \mathrm{g} \mathrm{h} \mathrm{L}^{-1}\right)$ and $\mathrm{MRT}_{0-t}(\mathrm{~h})$ of GPA were $1147.75 \pm 307.23,2119.16 \pm$ 530.84 and $4.10 \pm 0.33$ at a dose of $105 \mathrm{mg} \mathrm{kg}^{-1}$, and $3673.10 \pm 1250.44,8734.07 \pm 2362.48$ and 3.95 \pm 0.82 at a dose of $350 \mathrm{mg} \mathrm{kg}^{-1}$. The above pharmacokinetic parameters were higher than those of GP at the same dosage, which showed that GPA was likely to be the main absorbed saponin in vivo. These findings provide useful information that will support the studies on identification of bioactive platycosides in vivo, and will lay the foundation for the development of Jiegeng.

\section{Introduction}

Platycodi radix (Jiegeng), the dried root of Platycodon grandiflorum A. DC. (Campanulaceae), has been widely used as an antitussive or anti-inflammatory medicine in China and East Asia. ${ }^{1-3}$ In addition, Jiegeng has been reported to have many other pharmacological activities, such as anti-oxidant, ${ }^{\mathbf{4 , 5}}$ antitumor, ${ }^{6-9}$ anti-diabetic, ${ }^{10}$ anti-obesity, ${ }^{11,12}$ hepato-protective ${ }^{13,14}$ and cardiovascular system activities. ${ }^{\mathbf{1 5 , 1 6}}$ All the above activities are due to the chemical constituents in Jiegeng, including polysaccharides, saponins, flavonoids, polyphenols, polyacetylenes and sterols. ${ }^{1}$ The saponins in Jiegeng are also called platycosides, which have been proved to be the major bioactive compounds. ${ }^{17}$ Currently, all known platycosides are oleananetype pentacyclic triterpenoid saponins, which are mostly primary saponins. ${ }^{\mathbf{1 8}}$ These platycosides primarily have two saccharide chains; one or two glucoses linked through linear

${ }^{a}$ State Key Laboratory of Medicinal Chemical Biology, College of Pharmacy, Tianjin Key Laboratory of Molecular Drug Research, Nankai University, Tianjin 300353, People's Republic of China. E-mail: houyy@nankai.edu.cn

${ }^{b}$ State Key Laboratory of Quality Research in Chinese Medicine, Macau Institute for Applied Research in Medicine and Health, Macau University of Science and Technology, Taipa, Macau, People's Republic of China

$\dagger$ Electronic supplementary information (ESI) available: Tables S1-S6 and Fig. S1-S9. See DOI: 10.1039/c7ra04814a glycosidic bonds at the C-3, and arabinose, rhamnose, xylose and apiose sequentially attached at C-28 by ester linkages. ${ }^{19}$ According to the different aglycones, they are classified into four types, including paltcodic acid, polygalic acid, platcogenic acid and its derivatives. ${ }^{\mathbf{1}}$

Although there are a large number of studies about the activities of platycosides at present, there are only a few studies on the pharmacokinetics of platycosides. The pharmacokinetic studies of platycodin D, platycodin D3 and deapioplatycodin D have shown that these platycosides have low oral bioavailability, which due to the poor permeability and degradation in the gastrointestinal tract. ${ }^{20-25}$ In that case, we consider that activities of Jiegeng could be caused by the metabolites of platycosides or other absorbed platycosides.

In this study, the total saponins were isolated from the platycodi radix extract, and the main platycosides were identified by the ultra high pressure liquid chromatography along with quadrupole time of flight mass spectrometry (UPLC-Q/TOF-MS). According to the result of identification based on the fragmentation behaviors of platycosides, the absorbed saponins were qualitatively analyzed by principal components analysis (PCA). Then, the secondary platycosides, 3-O- $\beta$-D-glucopyranosylplatycodigenin (GP) and 3-O- $\beta$-D-glucopyranosylplatyconic acid (GPA), with higher relative contents were isolated and identified. And their pharmacokinetic parameters 
were evaluated using the liquid chromatography along with triple quadrupole mass spectrometry (LC-QQQ). By this way, we hoped to utilize the information about metabolites of platycosides to discover the bioactive constituents in vivo, and provide pharmacokinetic data support for subsequent pharmacological experiments.

\section{Materials and methods}

\subsection{Chemicals, reagents and animals}

Platycodi radix extract (bath number TR20160103) was purchased from Tianrui Bio-Tech Co., Ltd (Xi'an, China). Acetonitrile (HPLC grade) and methanol (HPLC grade) were purchased from Merck (Darmstadt, Germany). Ultrapure water was prepared with a Milli-Q system (Millipore, Bedford, MA, USA). All other reagents were analytical grade and purchased from Concord Technology (Tianjin, China). Ginsenoside Rh1 (98\% purity) was purchased from the Shilan Technology (Tianjin, China).

Experimental animals were purchased from the Laboratory Animal Center of the Academy of Military Medical Sciences (SCXK 2012-0004, Beijing, China). The animals were housed under standard laboratory conditions $\left(25 \pm 1{ }^{\circ} \mathrm{C}, 50 \pm 10 \%\right.$ humidity, and $12 \mathrm{~h}$ photoperiod) with free access to food and water. The animal experiments were approved by the Animal Experiments Ethical Committee of Nankai University and complied with the Guide for the Care and Use of Laboratory Animals. The animals were fasted $12 \mathrm{~h}$ and had free access to water throughout the experimental period.

\subsection{Instrumentation and conditions}

The UPLC-Q/TOF-MS system consisted of a Waters Acquity UPLC System (Waters Co., Milford, MA, USA) and a Waters Q/ TOF Premier instrument with an electrospray ionization system (Waters MS Technologies, Manchester, UK). An Acquity BEH C18 column $(2.1 \mathrm{~mm} \times 100 \mathrm{~mm}, 1.7 \mu \mathrm{m}$; Waters Co. $)$ was used with a mobile phase of $0.1 \%$ formic acid (A)-acetonitrile (B). The elution gradient was as follows: $0 \mathrm{~min}, 5 \% \mathrm{~B} ; 2 \mathrm{~min}$, $15 \%$ B; $18 \mathrm{~min}, 35 \%$ B. $23 \mathrm{~min}, 55 \%$ B; $27 \mathrm{~min}, 100 \%$ B; $29 \mathrm{~min}$ $100 \% \mathrm{~B}$. The flow rate was $0.40 \mathrm{~mL} \min ^{-1}$ and the column temperature was maintained at $30{ }^{\circ} \mathrm{C}$. The injection volume of the test sample was $5 \mu \mathrm{L}$. Data were acquired using MassLynx V4.1 software (Waters Co.). The capillary voltage was set to 2.0 $\mathrm{kV}$ for the negative mode and $3.0 \mathrm{kV}$ for the positive mode, and the sample cone voltage was set to $40 \mathrm{~V}$. The desolvation gas was set to $800 \mathrm{~L} \mathrm{~h}^{-1}$ at a temperature of $450{ }^{\circ} \mathrm{C}$, the cone gas was set to $50 \mathrm{~L} \mathrm{~h}^{-1}$, and the source temperature was $120{ }^{\circ} \mathrm{C}$. The mass spectrometric data were collected in full scan mode, and the $\mathrm{m} / \mathrm{z}$ ranged from 50 to $1900 \mathrm{Da}$ in the positive and negative ion modes.

The LC-QQQ platform was comprised of an Agilent 1290 infinity ultra-high performance liquid chromatography (UHPLC) system (Agilent Technologies, Santa Clara, USA) interfaced to an Agilent 6490 Triple Quadrupole LC/MS System (Agilent Technologies, Santa Clara, USA), using a standard-flow ESI (Jet Stream) source. The LC system specifically consists of a binary pump, a thermostatted column compartment, and a thermostatted micro well-plate autosampler. The column was an Acquity BEH C18 column $(2.1 \mathrm{~mm} \times 100 \mathrm{~mm}, 1.7 \mu \mathrm{m}$; Waters Co.), and the mobile phase consisted of $0.1 \%$ formic acid (A) and acetonitrile (B) using a gradient elution program as follows: $0 \mathrm{~min}, 30 \% \mathrm{~B}$; $8 \mathrm{~min}, 35 \% \mathrm{~B} ; 10 \mathrm{~min}, 85 \%$ B. $10.1 \mathrm{~min}$, $100 \% \mathrm{~B} ; 12 \mathrm{~min}, 100 \% \mathrm{~B}$. The column temperature was set to $25{ }^{\circ} \mathrm{C}$, the flow rate was maintained at $0.35 \mathrm{~mL} \mathrm{~min}^{-1}$, and the injection volume was set at $2 \mu \mathrm{L}$. All data are processed with the Agilent MassHunter Quantitative Analysis software (version B.06.00, Agilent Technologies). The capillary voltage was set to $4.0 \mathrm{kV}$ for the positive mode, and the fragmentor voltage was $250 \mathrm{~V}$. The sheath gas was set to $16 \mathrm{~L} \mathrm{~min}^{-1}$ at a temperature of $250{ }^{\circ} \mathrm{C}$. The nebulizer gas pressure was $35 \mathrm{psi}$.

\subsection{Metabolite identification of the total saponins}

2.3.1. Preparation of the total saponins. The powder of platycodi radix extract $(600 \mathrm{~g})$ was precipitated with $70 \%$ ethanol $(2 \mathrm{~L})$. After filtration, the supernatants were evaporated under reduced pressure. The extract was chromatographed over a macroporous resin $\mathrm{AB}-8$ column and eluted in accordance with the order of water, $30 \%$ ethanol, and $70 \%$ ethanol. The $70 \%$ ethanol fraction was collected, evaporated and lyophilised to obtain total saponins $(5.14 \mathrm{~g})$. The powder of total saponins was prepared into $50 \%$ methanol solution with a concentration of $3 \mathrm{mg} \mathrm{mL}^{-1}$ and filtered through a $0.22 \mu \mathrm{m}$ filter before the UPLC-Q/TOF-MS analysis.

2.3.2. Metabolite identification of the total saponins in vivo. Ten male $\mathrm{SD}$ rats were divided into experimental and control group randomly. In the experimental group, total saponins were administered orally at a dose of $600 \mathrm{mg} \mathrm{kg}$ body weight, and the control group was given physiological saline in the same manner. After $30 \mathrm{~min}$ of administration, the rats were anesthetized by $10 \%$ chloral hydrate $(0.3 \mathrm{~mL} / 100 \mathrm{~g})$, and the blood samples were collected in heparinized tubes and centrifuged to separate the plasma at $3000 \mathrm{rpm}$ for $10 \mathrm{~min}$ at $4{ }^{\circ} \mathrm{C}$. All the plasma samples were stored at $-80{ }^{\circ} \mathrm{C}$ until analysis.

The thawed plasma samples $(500 \mu \mathrm{L})$ were precipitated protein with methanol $(1.5 \mathrm{~mL})$. After vortexing for 2 min and centrifuging at $13000 \mathrm{rpm}$ for $10 \mathrm{~min}$ at $4{ }^{\circ} \mathrm{C}$, the supernatants were transferred to another tube and evaporated to dryness by $\mathrm{N}_{2}$. The residues were dissolved in methanol $(150 \mu \mathrm{L})$ and then centrifuged again at the same manner before the UPLC-Q/TOFMS analysis. The metabolism data were analyzed by principal components analysis (PCA) using SIMCA-P software (version 13.0, UmetricsAB, Sweden).

\subsection{Pharmacokinetic study of GP and GPA}

2.4.1. The isolation of GP and GPA. The powder of total saponins was separated by silica gel column in a gradient manner (EtOAc : $\mathrm{MeOH}=9: 1-1: 1$ ) to give five fractions (fr. A$\mathrm{E})$. Fraction $\mathrm{B}$ and $\mathrm{C}$ were collected and further separated by semipreparative HPLC (ACN : $\left.\mathrm{H}_{2} \mathrm{O}=35: 65\right)$ to get GP. GPA, the new compound, was obtained by the same method from fraction D. 
2.4.2. Preparation of calibration standard and quality control (QC) samples. The mixed stock solution was obtained by mixing the accurately weighed GP and GPA methanol stock solution, and given a final concentration of $499.20 \mu \mathrm{g} \mathrm{mL}{ }^{-1}$ for GP, $494.00 \mu \mathrm{g} \mathrm{mL} \mathrm{m}^{-1}$ for GPA. Then the mixed solution was diluted with methanol to obtain working solutions with desired concentrations. The concentration of ginsenoside Rh1 (internal standard, IS) solution was $2 \mu \mathrm{g} \mathrm{mL}{ }^{-1}$. All the solutions were stored at $4{ }^{\circ} \mathrm{C}$ and brought to room temperature before use.

Calibration standards and quality control (QC) samples were prepared as the following: working solution $(25 \mu \mathrm{L})$ was evaporated to dryness by $\mathrm{N}_{2}$, and then blank plasma $(100 \mu \mathrm{L})$ was added. The samples were prepared prior to the validation and pharmacokinetic study. The standards and QC samples were extracted on each analysis day with the same procedures for plasma samples as described below.

2.4.3. Preparation of the plasma samples. The IS solutions $(25 \mu \mathrm{L})$ were added into the plasma samples $(100 \mu \mathrm{L})$, and precipitated protein with methanol $(275 \mu \mathrm{L})$. After vortexing for $2 \mathrm{~min}$ and centrifuging at $13000 \mathrm{rpm}$ for $10 \mathrm{~min}$ at $4{ }^{\circ} \mathrm{C}$, the supernatants were transferred to another tube and evaporated to dryness by $\mathrm{N}_{2}$. The residues were redissolved in methanol $(100 \mu \mathrm{L})$ and centrifuged again at the same manner. Subsequently, the supernatants were transferred into vials for injection.

2.4.4. Method validation. The specificity of the method was evaluated by comparing the chromatograms of blank plasma samples, plasma samples spiked with the analytes and IS, and plasma samples after an oral dose. The calibration curves were plotted by the peak-area ratios $(y)$ of analytes against IS $v s$. the nominal concentration $(x)$. The precision and accuracy were determined from intra- and inter-batch analysis at three different QC levels, and expressed as relative standard deviation (RSD) and relative error (RE), respectively. The extraction recoveries were determined by comparing the peak areas obtained from three QC samples with those obtained from postextracted blank plasma with the pure reference standards at the same concentration. The latter peak areas compared to those obtained from the pure reference standard solutions at the same concentration for evaluation of the matrix effects. The stability was assessed using QC samples under different storage conditions: short-term stability at room temperature for $24 \mathrm{~h}$, long-term stability at $-20{ }^{\circ} \mathrm{C}$ for 14 days, and post-preparation stability at $4{ }^{\circ} \mathrm{C}$ for $24 \mathrm{~h}$. Three freeze-thaw cycles $\left(-20{ }^{\circ} \mathrm{C}\right.$ to room temperature) were used for evaluation of the freeze-thaw stability on consecutive days.

2.4.5. Pharmacokinetic study. The male Kunming mice were randomly divided into two groups ( $n=6$ at each point). One group was administered total saponins orally at a dose of $105 \mathrm{mg} \mathrm{kg}^{-1}$ and the other was at $350 \mathrm{mg} \mathrm{kg}^{-1}$. Blood samples were collected in heparinized tubes at $0,0.25,0.5,0.75,1,1.5,2$, 4,8 and $12 \mathrm{~h}$ after oral administration and centrifuged to separate the plasma at $3000 \mathrm{rpm}$ for $10 \mathrm{~min}$ at $4{ }^{\circ} \mathrm{C}$. All the plasma samples were stored at $-80{ }^{\circ} \mathrm{C}$ until analysis. The pharmacokinetic parameters, including the peak plasma concentration $\left(C_{\max }\right)$, the time to $C_{\max }\left(T_{\max }\right)$, the AUC from 0 to infinity $\left(\mathrm{AUC}_{0-\infty}\right)$, the AUC form 0 to time $\left(\mathrm{AUC}_{0-t}\right)$, the mean residence time (MRT) and the terminal elimination half-life $\left(T_{1} /\right.$ ${ }_{2 z}$ ) were calculated by the non-compartmental analysis of plasma concentration $v s$. time data using the "DAS 2.1.1" software (Mathematical Pharmacology Professional Committee of China, Shanghai, China).

\section{Results}

\subsection{Qualitative analysis of the components of total saponins}

The optimal UPLC-Q/TOF-MS conditions were applied for the analysis of the total saponins. More ion information of platycosides was obtained from positive ion mode, so the positive ion mode was selected in the following investigations (Fig. 1).

In positive mode, prominent peaks were mostly contained protonated platycoside molecules and their corresponding secondary saponin molecules. Taking the identify process of peak 5 as an example, it gave $[\mathrm{M}+\mathrm{H}]^{+}$ion at $m / z 1225$ in MS that presumed as platycodin D. Meanwhile it generated the prominent ions at $m / z 1093,961,683$ and 521 as base peaks in the MS/MS by sequential lost apiosyl, xylosyl, rhamosyl, and arabinosyl residues linked at C-28, and loss of glucosyl residues linked at C-3 of the triterpene, respectively. The above rule and structure were consistent with literature of platycodin $\mathrm{D},{ }^{26}$ so the peak 5 was identified as platycodin D. According to this process, peak 1, 2, 4, 5 and 7-12 were identified.

The above method was suitable for the identification of known platycosides, meanwhile the unknown compounds using reverse ideas for identifying. Taking peak 13 as the example, the prominent ion at $m / z 535$ was found in the positive $\mathrm{MS} / \mathrm{MS}$, and the $m / z$ of $[\mathrm{M}+\mathrm{H}]^{+}$ion in MS was 697, which proved
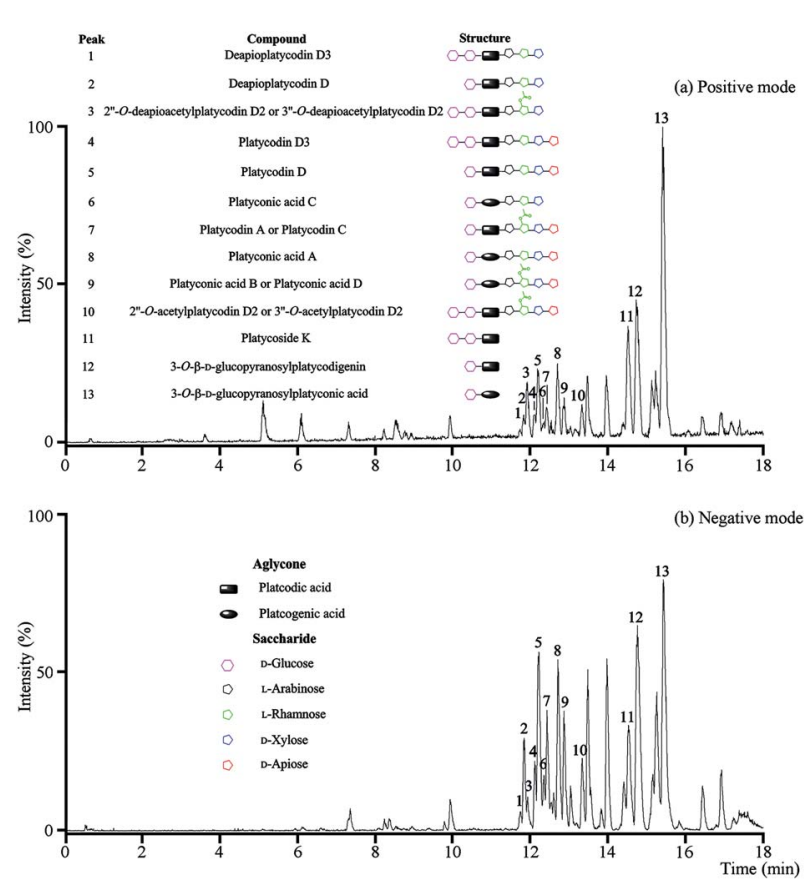

Fig. 1 UPLC-Q/TOF-MS analysis of the total saponins. (a) The BPI chromatogram in positive mode; (b) the BPI chromatogram in negative mode. 
this compound was platcogenic acid-type platycoside with the exact molecular weight at 696.3615. According to the fragmentations and structural characteristics, this compound was presumed as a monodesmosidic platycoside with a glucosyl at C-3 named 3-O- $\beta$-D-glucopyranosylplatyconic acid. The identification of peak 3 was similar. These two platycosides were reported for the first time, which would be separated and further confirmed in the subsequent studies if necessary.

These methods were applicable for the fast and accurate identification of known and unknown platycosides. However, isomeric platycosides and constituents present at low levels were unidentifiable.

As a result, thirteen platycosides that respectively belonged to paltcodic acid- and platcogenic acid-type were identified accurately and effectively (Table $\mathrm{S} 1 \dagger$ ). The structures of these platycosides were described in Fig. S1. $\dagger$

\subsection{Qualitative analysis of total saponins constituents in vivo}

The PCA score plots (Fig. 2a) presented the distribution among experimental and control group based on the metabolic profiles. Loading plots (Fig. 2b) indicated that markers made greater contribution to separation with farther away from original point, and the markers with the same retention time might be from the same compound.

According to the retention time and $\mathrm{m} / \mathrm{z}$ of markers, nineteen markers were identified belong to seven absorbed platycosides (Table 1 and Fig. S1†), which were significantly altered in the experimental group compared to the control group.

An extracted ion chromatogram (EIC) strategy was established to capture platycosides and determine their relative contents in plasma samples according to the characteristic ion pairs. The ion pair at $\mathrm{m} / \mathrm{z} 521$ and 683 was selected as the diagnostic ions for paltcodic acid-type platycosides, and platcogenic acid-type platycosides used $\mathrm{m} / \mathrm{z} 535$ and 697 as characteristic ions. The EICs (Fig. 2c and d) were shown that the relative contents of GP and GPA were higher than the primary platycosides in rat plasma after oral administration of total saponins for 30 min. As a result, GP and GPA were determined as target compounds in the subsequent quantitative analysis in vivo.

\subsection{Separation and structure identification of GP and GPA}

GPA (Fig. 3) was an amorphous powder and the molecular formula was assigned to be $\mathrm{C}_{36} \mathrm{H}_{56} \mathrm{O}_{13}$ based on the highresolution spectrum. The spectral features and physicchemical properties revealed it to be a triterpenoid saponin.

The ${ }^{1} \mathrm{H}$ NMR spectrum (pyridine- $d_{5}, 600 \mathrm{MHz}$ ) showed five tertiary methyl groups $(\delta 1.05,1.09,1.18,1.65$ and 1.84) and one olefinic proton $\left(\delta 5.66\right.$, br s). The ${ }^{13} \mathrm{C}$ NMR spectrum (pyridine- $d_{5}$, $600 \mathrm{MHz}$ ) showed five $\mathrm{sp}^{3}$ carbons at $\delta 15.64,17.43,24.72,27.28$ and 33.37, two $\mathrm{sp}^{2}$ olefinic carbons at $\delta 122.41$ and 145.30 , two carboxyl carbons at $\delta 176.31$ and 179.99, and four oxygenated methene and methine carbons at $\delta 69.97,81.19,74.72$ and 62.67. The information of ${ }^{1} \mathrm{H}$ and ${ }^{13} \mathrm{C}$ NMR spectrum indicated that it possessed a 2,3,16,23-tetrahydroxyolean-12-ene-24,28-dioic acid as aglycon. The chemical shifts of C-3 $(\delta$ 81.19) and C-28 $(\delta$ 179.99) revealed that it was a monodesmosidic glycoside. The ${ }^{1} \mathrm{H}$ and ${ }^{13} \mathrm{C}$ NMR spectra of it exhibited one sugar anomeric proton at $\delta 5.40(1 \mathrm{H}, \mathrm{d} . J=7.8 \mathrm{~Hz})$, and carbon at $\delta 106.70$. On the basis of the above evidence, 3-O- $\beta$-D-glucopyranosyl-platyconic acid was identified to be $3-O-\beta$-D-glucopyranosyl-2,3,16,23-
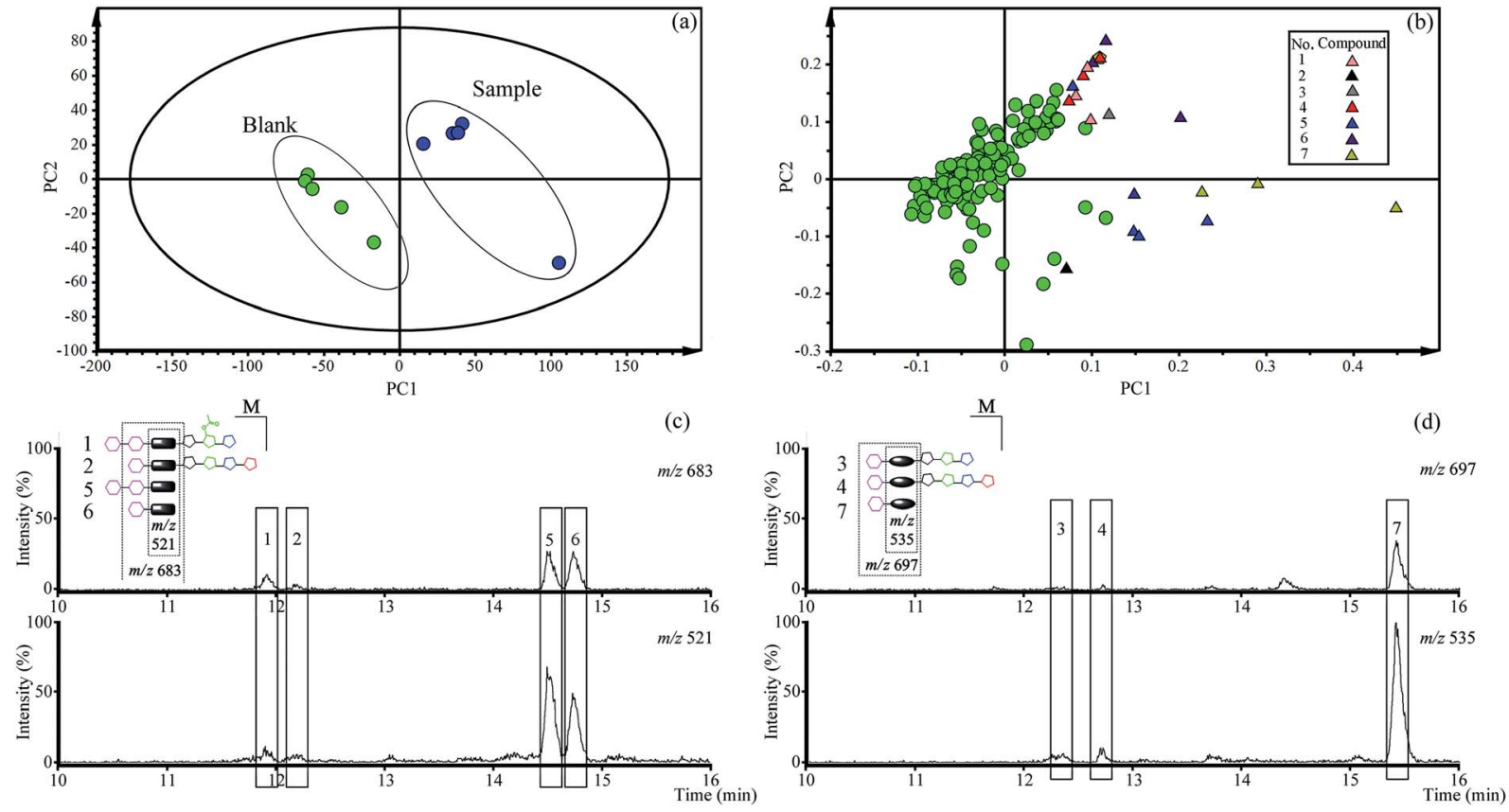

Fig. 2 The results of qualitative analysis of total saponins in vivo by PCA. (a) Score plotting; (b) loading plotting; (c) the extracted ion chromatograms of paltcodic acid-type platycosides; (d) the extracted ion chromatograms of platcogenic acid-type platycosides. 
Table 1 Seven markers identified in plasma samples by UPLC-Q/TOF-MS with PCA

\begin{tabular}{|c|c|c|c|c|}
\hline No. & $\begin{array}{l}\text { Ret. time } \\
\text { (min) }\end{array}$ & Marker & Formula & Compound \\
\hline 1 & 11.94 & $845,683,521$ & $\mathrm{C}_{60} \mathrm{H}_{96} \mathrm{O}_{30}$ & $2^{\prime \prime}$-O-Deapioacetylplatycodin D2 \\
\hline 3 & 12.32 & 517 & $\mathrm{C}_{65} \mathrm{H}_{104} \mathrm{O}_{34}$ & Platyconic acid C \\
\hline 4 & 12.74 & $1107,975,535$ & $\mathrm{C}_{57} \mathrm{H}_{90} \mathrm{O}_{29}$ & Platyconic acid A \\
\hline 5 & 14.55 & $867,845,683,521,503$ & $\mathrm{C}_{42} \mathrm{H}_{68} \mathrm{O}_{17}$ & Platycoside $\mathrm{K}$ \\
\hline
\end{tabular}

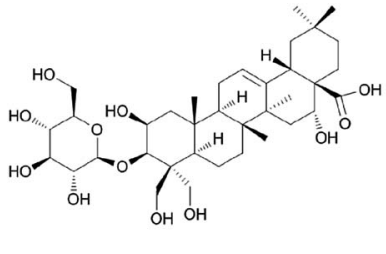

GP

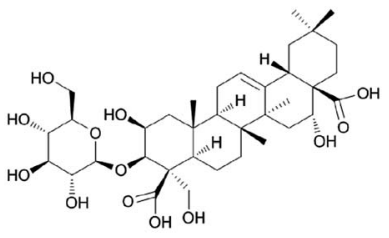

GPA

Fig. 3 Structures of GP and GPA.

tetrahydroxyolean-12-ene-24,28-dioic acid. Other NMR spectra data were described in Fig. S2-S7 and Table S2. $\dagger$

GP (Fig. 3) was identified by comparing with the spectra data reported in the literature $\left({ }^{1} \mathrm{H}\right.$ and ${ }^{13} \mathrm{C}$ NMR are described in Table S2 and Fig. S8 and S9†). ${ }^{27,28}$

\subsection{Method validation for quantitative analysis}

The optimized MRM modes were applied for the analysis (Table S3 $\dagger$ ). No obvious endogenous interference was observed when analytes and IS were detected, and the different retention times and molecule polarity were used to distinguish platycosides that produced the same fragment ions in the plasma samples (Fig. 4).

All calibration curves exhibited good linearity $\left(r^{2} \geq 0.9995\right)$. Extraction recoveries and matrix effects of analytes and IS were in the range of $91.96-99.94 \%$ (RSD $\leq 5.40 \%$ ) and 93.45$111.65 \%$ (RSD $\leq 8.22 \%$ ), respectively. RSD of intra-and interbatch precisions were in the range of $1.19-12.28 \%$ and 1.09 $6.92 \%$, and RE from -1.18 to $9.52 \%$, respectively (Table 2 ). The detected concentrations for all analytes were in the range of -11.37 to $6.61 \%$ of original values, indicating that they were stable in plasma samples after three freeze-thaw cycles, at room temperature and autosampler for $24 \mathrm{~h}$, at $-20{ }^{\circ} \mathrm{C}$ for 14 days (partial data were described in Tables S4-S6†).

\subsection{Pharmacokinetic study}

The validated method described above was successfully applied to the pharmacokinetic study in which plasma concentrations of two analytes were determined for $12 \mathrm{~h}$ after the oral administration of total saponins (105 $\mathrm{mg} \mathrm{kg}^{-1}$ and $350 \mathrm{mg} \mathrm{kg}^{-1}, n=6$ ). The mean plasma concentration time profiles of all analytes (Fig. 5) revealed some similarities in concentration-time curve for them, and the main pharmacokinetic parameters were exhibited in Table 3. All analytes were quickly absorbed within $1 \mathrm{~h}$, and a low peak appeared in the curve due to the enterohepatic circulation at approximately $1.5 \mathrm{~h}$ after oral administration. And the five important pharmacokinetic parameters, including $T_{1 / 2 z}(\mathrm{~h}), \mathrm{AUC}_{0-t}\left(\mu \mathrm{g} \mathrm{h} \mathrm{L}{ }^{-1}\right), \mathrm{AUC}_{0-\infty}\left(\mu \mathrm{g} \mathrm{h} \mathrm{L}{ }^{-1}\right), \mathrm{MRT}_{0-t}$ (h) and $\mathrm{MRT}_{0-\infty}(\mathrm{h})$, for GP, were lower than those of GPA at both doses of total saponins. It's worth noting that the $C_{\max }\left(\mu \mathrm{g} \mathrm{L}^{-1}\right)$ of GPA with the dosage of $350 \mathrm{mg} \mathrm{kg}^{-1}$ total saponins reached $3673.10 \pm 1250.44 \mu \mathrm{g} \mathrm{L}^{-1}$, which was a high concentration of the drug in the plasma. Pharmacokinetic study results also showed that metabolic behaviors were similar for two secondary saponins, which made sense that GP and GPA had similar metabolic regularity. By comparing the pharmacokinetic parameters and

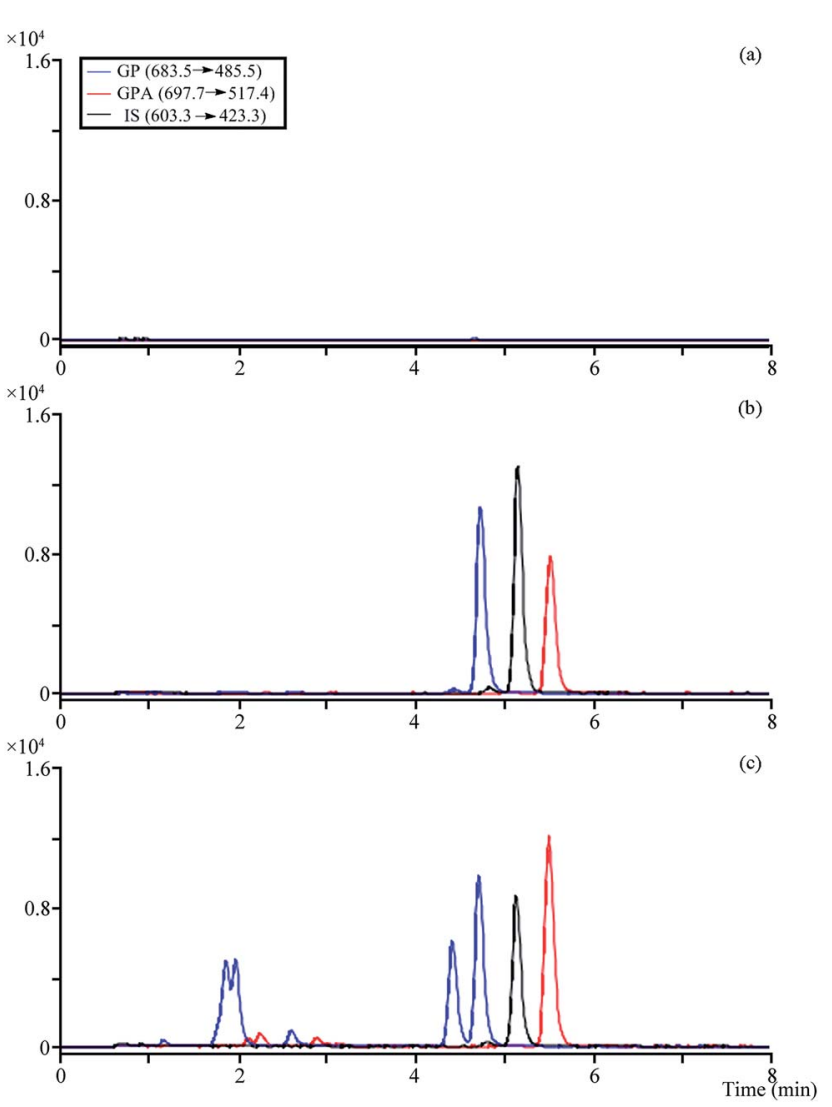

Fig. 4 The MRM chromatograms of analytes and IS. (a) Blank plasma; (b) blank plasma spiked with the analytes and IS; (c) $0.5 \mathrm{~h}$ after administration of plasma sample. 
Table 2 Inter-and intra-batch precision and accuracy data of analytes in plasma samples (mean $\pm \mathrm{SD}, n=6$ )

\begin{tabular}{|c|c|c|c|c|c|}
\hline \multirow[b]{2}{*}{ Analytes } & \multirow[b]{2}{*}{$\begin{array}{l}\text { Conc. } \\
\left(\mu \mathrm{g} \mathrm{L}^{-1}\right)\end{array}$} & \multicolumn{2}{|c|}{ Inter-batch } & \multicolumn{2}{|c|}{ Intra-batch } \\
\hline & & $\begin{array}{l}\text { Precision } \\
\text { (RSD, \%) }\end{array}$ & $\begin{array}{l}\text { Accuracy } \\
\text { (RE, \%) }\end{array}$ & $\begin{array}{l}\text { Precision } \\
\text { (RSD, \%) }\end{array}$ & $\begin{array}{l}\text { Accuracy } \\
(\%)\end{array}$ \\
\hline \multirow[t]{3}{*}{ GP } & 2.47 & 3.93 & 5.51 & 4.42 & -0.74 \\
\hline & 247.00 & 1.19 & 1.43 & 1.09 & 6.87 \\
\hline & 2470.00 & 2.81 & 4.80 & 2.66 & 9.52 \\
\hline & 2496.00 & 6.35 & 5.07 & 6.92 & 6.03 \\
\hline
\end{tabular}
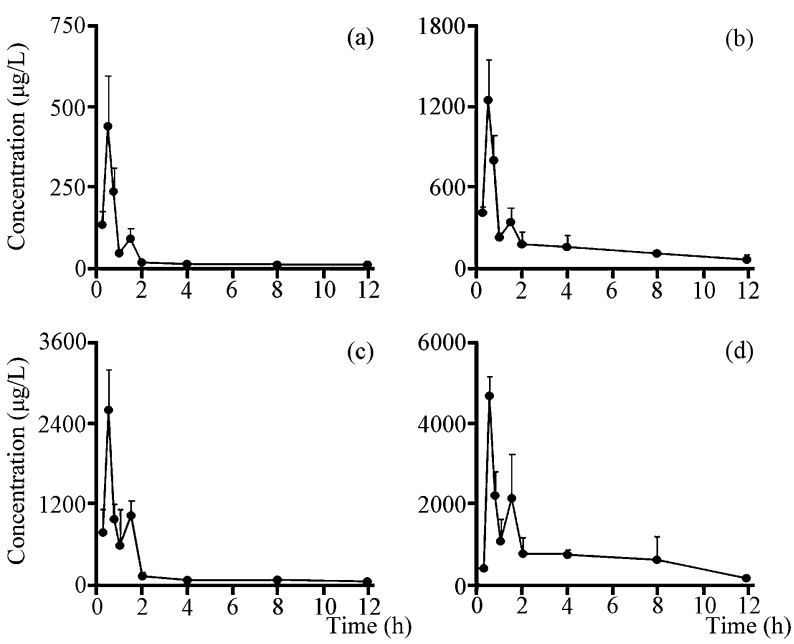

Fig. 5 Mean concentration-time profiles of GP and GPA in mice plasma after oral administration of total saponins. (a) GP (105 mg kg$\left.{ }^{-1}\right)$; (b) GPA (105 mg kg ${ }^{-1}$ ); (c) GP (350 $\left.\mathrm{mg} \mathrm{kg}^{-1}\right)$; (d) GPA (350 $\left.\mathrm{mg} \mathrm{kg}^{-1}\right)$.

metabolic regularity, GPA was considered to be the main absorbed platycoside in vivo.

\section{Discussion}

Jiegeng as the representative medicine of meridian-guiding theory has been shown to promote the absorption and targeted distribution of other drugs in the lungs. ${ }^{29-31}$ Although the mechanism is not yet clear, the platycosides are considered to be the main active ingredients due to the amphiphilicity. ${ }^{17,32-34}$ In this study, thirteen platycosides were identified in the total saponins that isolated from the root of platycodi radix extract. Among these saponins, ten saponins belonged to primary saponins and other three were secondary saponins.

We found $T_{\max }$ of platycodin D and platycodin D3 were both less than $1 \mathrm{~h}$ after oral administration to rats in the previous pharmacokinetic studies, ${ }^{\mathbf{2 1 - 2 3 , 2 5}}$ therefore we selected $30 \mathrm{~min}$ as a critical time point in metabolic qualitative research of total saponins. PCA was used qualitatively analyze the metabolites of Jiegeng in vivo, the score plots proved a significant distinction between the experimental and control group. By analyzing the contribution value, $\mathrm{m} / \mathrm{z}$ and retention time of markers in loading plots, nineteen markers were identified from seven absorbed platycosides.

Although thirteen platycosides were identified in total saponins, only seven among these were confirmed to contribute to the discrimination between experimental and control group. The result demonstrated that most of primary platycosides may be degraded or not absorbed in gastric-intestinal tract, which was in accordance with the previous reports. ${ }^{20,24}$

Furthermore, some differences between the metabolites in vitro and in vivo were found. The metabolites of platycosides in vivo all contained glucoses at C-3, but in vitro the glucoses could be easily hydrolyzed by enzyme that produced by intestinal bacteria; the secondary platycosides without saccharide chain at C-28 were the main absorbed compounds in vivo, but the saccharide chain at C-28 cloud not be enzymatic hydrolyzed in vitro and the main metabolites of platycosides in vitro were the compounds with the acetylated saccharide at C-28. ${ }^{17,24,35}$ So the hydrolysis of saccharide chain at C-28 by gastric acid in the

Table 3 The pharmacokinetic parameters of GP and GPA in mice plasma (mean \pm SD, $n=6$ )

\begin{tabular}{|c|c|c|c|c|}
\hline \multirow[b]{2}{*}{ Parameters } & \multicolumn{2}{|l|}{$105 \mathrm{mg} \mathrm{kg}^{-1}$} & \multicolumn{2}{|l|}{$350 \mathrm{mg} \mathrm{kg}^{-1}$} \\
\hline & GP & GPA & GP & GP \\
\hline $\operatorname{AUC}_{0-\infty}\left(\mu \mathrm{g} \mathrm{h} \mathrm{L}{ }^{-1}\right)$ & $353.77 \pm 55.56$ & $2391.75 \pm 547.42$ & $2436.92 \pm 421.65$ & $9446.18 \pm 2426.74$ \\
\hline $\mathrm{MRT}_{0-t}(\mathrm{~h})$ & $2.23 \pm 0.42$ & $4.10 \pm 0.33$ & $2.16 \pm 0.25$ & $3.95 \pm 0.82$ \\
\hline $\mathrm{MRT}_{0-\infty}(\mathrm{h})$ & $3.36 \pm 0.81$ & $5.76 \pm 0.84$ & $3.02 \pm 0.76$ & $4.97 \pm 0.79$ \\
\hline$C_{\max }\left(\mu \mathrm{g} \mathrm{L}^{-1}\right)$ & $392.61 \pm 127.71$ & $1147.75 \pm 307.23$ & $2434.17 \pm 818.94$ & $3673.10 \pm 1250.44$ \\
\hline
\end{tabular}


stomach was considered to be the main reason of the degradations of primary platycosides and the differences between the metabolites in vitro and in vivo.

By comparing the relative contents obtained from peak areas in EIC, and considering the absorption and degradation of saponins, the two secondary saponins with a glucosyl linked at C-3, including GP and GPA, were used as the representative compounds in pharmacokinetic analysis.

The result of pharmacokinetic study showed that GP and GPA reached $C_{\max }$ within $1 \mathrm{~h}$ after administration in dosage of high and low, which means the rapid absorption and acting in vivo. Although the contents of GP and GPA in the total saponins were $1.03 \%$ and $0.45 \%$ respectively, the main important pharmacokinetic parameters of GP were lower than GPA. The reason could be that the acidity of GPA is stronger than GP, and the absorption of acidic compounds in the stomach is better due to gastric acid $(\mathrm{pH}=1.2) .{ }^{36}$ On the other hand, this explains why secondary saponins containing carboxyl group at C-28 were more easily absorbed into blood than primary saponins with saccharide chain at C-28.

Based on the qualitative and quantitative results of metabolic analyses of Jiegeng in vivo, the reasons of secondary saponins as the main absorbed components are summarized as follows:

(a) The poor membrane permeability of primary saponins causes difficult to cross a biological barrier by active transport; (b) the acidic secondary saponins are easily absorbed in acidic environment of the stomach; (c) the primary saponins are hydrolysis to produce secondary saponins under the action of gastric acid. Although GPA was proved to be the most important absorbed platycoside in vivo, the further studies about the effect comparison between GP and GPA will be made in the future.

Besides a few studies on pharmacokinetics of platycodin $\mathrm{D}$ and D3, there have been no reports about the pharmacokinetics of the absorbed platycosides after the oral administration of Jiegeng. The results of metabolite identification and pharmacokinetics of the absorbed saponins of Jiegeng in vivo will help researchers explore the mechanism of meridian-guiding effect in the subsequent pharmacological studies.

\section{Conclusion}

This paper developed qualitative and quantitative analyses of bioactive saponins of Jiegeng in vivo. Using the method based on the fragmentation behaviors of platycosides, a total of thirteen platycosides were identified, and seven among these were found in rat plasma after oral administration of total saponins by PCA. A simultaneous quantitative method of GP and GPA, the major absorbed platycosides in mice plasma, was established and validated by LC-QQQ. And the pharmacokinetic parameters of the two platycosides in vivo were revealed. This investigation provided helpful information to clear about bioactive platycosides of Jiegeng in vivo, and provided pharmacokinetic data support for subsequent pharmacological researches.

\section{Acknowledgements}

This work was supported by the National Natural Science Foundation of China (81374046, 81473403 and 81673616).

\section{Notes and references}

1 L. Zhang, Y. L. Wang, D. W. Yang, C. H. Zhang, N. Zhang, M. H. Li and Y. Z. Liu, J. Ethnopharmacol., 2015, 164, 147161.

2 T. W. Kim, J. H. Lim, H. K. Lee, M. Giorgi, H. Owen and H. I. Yun, Med. Weter., 2014, 70, 38-41.

3 Y. Xie, H. Pan, H. Sun and D. Li, Vaccine, 2008, 26, 39373945.

4 C. H. Jeong, G. N. Choi, J. H. Kim, J. H. Kwak, D. O. Kim, Y. J. Kim and H. J. Heo, Food Chem., 2010, 118, 278-282.

5 X. J. Fu, H. B. Liu, P. Wang and H. S. Guan, Am. J. Chin. Med., 2009, 37, 967-975.

6 S. Zheng, W. Li, J. Wang, Y. Chen, W. Hou, W. Gao, Q. Liu and Y. Wang, RSC Adv., 2016, 6, 10606-10614.

7 L. J. Xiu, D. Z. Sun, J. P. Jiao, B. Yan, Z. F. Qin, X. Liu, P. K. Wei and X. Q. Yue, J. Ethnopharmacol., 2015, 172, 155-161.

8 M. Khan, A. Maryam, H. Zhang, T. Mehmood and T. Ma, J. Cell. Mol. Med., 2016, 20, 389-402.

9 N. H. Yim, Y. H. Hwang, C. Liang and J. Y. Ma, J. Ethnopharmacol., 2016, 194, 1060-1068.

10 D. Y. Kwon, Y. S. Kim, S. Y. Ryu, Y. H. Choi, M. R. Cha, H. J. Yang and S. Park, Eur. J. Nutr., 2012, 51, 529-540.

11 H. M. Park, K. T. Park, E. C. Park, S. I. Kim, M. S. Choi, K. H. Liu and C. H. Lee, Nutrients, 2017, 9, e71.

12 Y. P. Hwang, J. H. Choi, H. G. Kim, T. Khanal, G. Y. Song, M. S. Nam, H.-S. Lee, Y. C. Chung, Y. C. Lee and H. G. Jeong, Toxicol. Appl. Pharmacol., 2013, 267, 174-183.

13 J. H. Choi, S. W. Jin, H. G. Kim, T. Khanal, Y. P. Hwang, K. J. Lee, C. Y. Choi, Y. C. Chung, Y. C. Lee and H. G. Jeong, Food Chem. Toxicol., 2013, 56, 231-239.

14 W. Li, Y. Liu, Z. Wang, Y. Han, Y.-H. Tian, G.-S. Zhang, Y.-S. Sun and Y.-P. Wang, Food Funct., 2015, 6, 1418-1427.

15 J. T. Wu, G. W. Yang, W. X. Zhu, W. J. Wen, F. M. Zhang, J. D. Yuan and L. G. An, Biol. Pharm. Bull., 2012, 35, 12161221.

16 E. M. Twiner, Z. J. Liu, J. Gimble, Y. Yu and F. Greenway, Adv. Ther., 2011, 28, 857-865.

17 E. Nyakudya, J. H. Jeong, N. K. Lee and Y.-S. Jeong, Prev. Nutr. Food Sci., 2014, 19, 59-68.

18 L. F. Zeng, H. J. Kong, M. Zhu and W. D. Yan, J. Funct. Foods, 2016, 26, 48-56.

19 E. K. Jeong, I. J. Ha, Y. S. Kim and Y. C. Na, J. Sep. Sci., 2014, 37, 61-68.

20 J. W. Lee, S. H. Ji, G. S. Kim, K. S. Song, Y. Um, O. T. Kim, Y. Lee, C. P. Hong, D. H. Shin, C. K. Kim, S. E. Lee, Y. S. Ahn and D. Y. Lee, Int. J. Mol. Sci., 2015, 16, 2678626796.

21 T. H. Kim, B. E. Lee, E. J. Kim, Y. S. Choi, K. S. Lee, H. R. Kim and H. G. Kim, Sci. World J., 2014, 10, DOI: 10.1155/2014/ 231293. 
22 Q. Zhan, F. Zhang, S.-H. Gao, F. Cai, B. Jiang, L.-N. Sun and W.-S. Chen, Chin. J. Nat. Med., 2014, 12, 154-160.

23 J. Shan, J. Zou, T. Xie, A. Kang, W. Zhou, H. Deng, Y. Mao, L. Di and S. Wang, Pharmacogn. Mag., 2015, 11, 750-755.

24 Y. W. Ha, Y.-C. Na, I. J. Ha, D.-H. Kim and Y. S. Kim, J. Pharm. Biomed. Anal., 2010, 51, 202-209.

25 L. Pei, Y. Bao, L. Ma, Q. Wang, Y. Ye, X. Han, S. Liu and X. Chen, Planta Med., 2012, 78, 244-251.

26 Y. C. Na, Y. W. Ha, Y. S. Kim and K. J. Kim, J. Chromatogr. A, 2008, 1189, 467-475.

27 L. J. Li, Z. H. Liu, Y. Chen and J. K. Tian, China J. Chin. Mater. Med., 2006, 31, 1506-1509.

28 W. W. Fu, D. Q. Dou, W. B. Hou, B. H. Cheng, F. Liu, Y. J. Chen, Y. H. Pei and T. Takeda, China J. Chin. Mater. Med., 2005, 297-301.
29 L. Zhang, L. Zhu, Y. F. Wang, Z. Z. Jiang, X. Chai, Y. Zhu, X. M. Gao and A. D. Qi, J. Pharm. Biomed. Anal., 2012, 62, 203-209.

30 J. S. Yu, C. H. Ho, Y. C. Hsu, J. J. Wang and C. L. Hsieh, J. Integr. Med., 2014, 6, 538-544.

31 W. W. Tao, Q. Su, H. Q. Wang, S. Guo, Y. Y. Chen, J. N. Duan and S. M. Wang, Int. Immunopharmacol., 2015, 27, 138-147.

32 X. X. Dai, H. O. Ding, Q. Q. Yin, G. Wan, X. Y. Shi and Y. J. Qiao, J. Mol. Graphics Modell., 2015, 57, 20-26.

33 J. M. Augustin, V. Kuzina, S. B. Andersen and S. Bak, Phytochemistry, 2011, 72, 435-457.

34 J. H. Lorent, J. Quetin-Leclercq and M. P. Mingeot-Leclercq, Org. Biomol. Chem., 2014, 12, 8803-8822.

35 W. Zhang, S. H. Qian, D. W. Qian and S. L. Li, Am. J. Chin. Med., 2016, 44, 817-833.

36 Z. Liu, X. Zheng, Y. Guo, W. Qin, L. Hua and Y. Yang, Fitoterapia, 2016, 113, 27-34. 